\title{
Prevalence of Micro-Organisms Isolated from Ear Swab
}

\author{
Dr. Neeti Mishra ${ }^{1}$, Dr. Shailendra Mohan Tripathi ${ }^{2}$ \\ ${ }^{1}$ Assistant Professor Department of Microbiology, Hind Institute of Medical Sciences, Safedabad Lucknow, India
}

Assistant Professor Department of Geriatric Mental Health, KGMU Lucknow, India

\begin{abstract}
Introduction: Discharge from ear is one of the commonest symptoms of infection of the ear. To screen various pathogenic organisms isolated from the ear swab and to determine their antibiotic sensitivity pattern against the commonly used antibiotics. Materials and Methods: Ear swabs were collected from different patients and subjected to sensitivity test was performed. Result: A total 400 specimens were collected from different patients in which 230 specimens showed positive growth and 170 specimens showed no growth. Conclusions: This study demonstrated that Staphylococcus aureus and Pseudomonas aeruginosa is the commonest organisms, isolated from ear swab which causes of ear discharge. Ciprofloxacin and gentamycin are effective ototopic antimicrobial agents for emprial treatment of the discharge ear.
\end{abstract}

Keywords: Ear discharge , Antimicrobial drugs , antibiotics sensitivity pattern

\section{Introduction}

Ear infection is an inflammation of the ear and ear discharge is one of the commonest symptoms of ear infection [1]. About 65-330 million people suffer from ear infection worldwide and $60 \%$ of them had significant hearing impairment [2]. The health-economic burden of ear infection is also severe especially in Africa and other developing nations where the disease prevalence is estimated as high as $11 \%$ []ㅡ.

Ear infection is a common problem for both children and adults but the magnitude is different in different countries. Anatomically the children's Eustachian tube is shorter, more horizontal with a more flaccid cartilage which can easily impair its opening and hence ear infection is a major health problem of them especially in those with poor socioeconomic status [4].

Otitis media, an infection of the middle ear, is usually associated with upper respiratory tract infection. Although, it can occur at any age, but $60-80 \%$ of children infected with recurrent otitis media [6]. Early diagnosis may avoid the possibility of complications associated with such infection like, brain abscess, meningitis, lateral sinus thrombosis and subdural abscess [7]. The infection was attributed to many bacterial species like Staphylococcus aureus, Proteus mirabilis, Proteus vulgaris, and Pseudomonas aeruginosa $[8,9]$.

Chronic suppurative otitis media (CSOM) is a prevailing and notorious infection in developing countries causing serious local damage and threatening complications. It is characterized by painless discharge from ears dating from months to years and is the single major cause of deafness in India. It is common amongst children of low socio economic strata [5].

\section{Materials and Methods}

The study conducted on 230 isolates that obtained from ear swab submitted to the department of microbiology from
December 2015 to Febuary 2016, were included in the study. The swabs were inoculated on the Mac Conkey agar and Blood agar, these plates were incubated aerobically at $37^{\circ} \mathrm{C}$ for 24 to 48 hours. All plates which showed growth were processed as per standard microbiological procedures. Antibiotic sensitivity testing was done by Kirby Bauer disk diffusion method using Mueller-Hinton agar. [10]

\section{Result}

A total of 400 samples were included in the study, out of which 230 showed positive bacterial growth and 170 were negative for any bacterial growth.

Out of 230 isolates , 80 Staphylococus aureus , 10 Enterococcus species , 10 E. coli , 10 Klebsiella pneumonae, 70 Pseudomonas aeruginosa, 20 Citrobacter species, 30 Proteus spp. Staphylococus aureus and Pseudomonas aeruginosa showed maximum growth followed by other organisms .

Antibiotic sensitivity test of all the clinical isolates was performed as per CLSI (2015) and sensitivity pattern was noted.

Table 1: Total number of isolates in the ear swab culture

\begin{tabular}{|c|c|}
\hline Organisms & Number of Isolates \\
\hline Staphylococus aureus & $80(34.7 \%)$ \\
\hline Enterococcus species & $10(4.3 \%)$ \\
E. coli & $10(4.3 \%)$ \\
Klebsiella pneumonae & $10(4.3 \%)$ \\
\hline Pseudomonas aeruginosa & $70(30.4 \%)$ \\
\hline Citrobacter species & $20(8.6 \%)$ \\
\hline Proteus mirabilis & $30(13 \%)$ \\
\hline TOTAL & \multicolumn{2}{|c|}{230} \\
\hline
\end{tabular}




\section{International Journal of Science and Research (IJSR) \\ ISSN (Online): 2319-7064}

Index Copernicus Value (2013): 6.14 | Impact Factor (2014): 5.611

Table 2: Antibiotic sensitivity pattern of gram positive isolates

\begin{tabular}{|c|c|c|c|c|}
\hline \multirow{2}{*}{ Antibiotics } & \multicolumn{3}{|c|}{ ORGANISMS } & \multicolumn{2}{c|}{$(\mathrm{n}=90)$} \\
\cline { 2 - 5 } & \multicolumn{2}{|c|}{$\begin{array}{c}\text { Staphyloccus aureus } \\
\text { (n=80) }\end{array}$} & \multicolumn{2}{c|}{$\begin{array}{c}\text { Enterococcus } \\
\text { spp. }(\mathrm{n}=10)\end{array}$} \\
\cline { 2 - 5 } Amikacin & S & $\mathbf{R}$ & $\mathbf{S}$ & $\mathbf{R}$ \\
Clindamycin & - & 10 & 6 & 4 \\
\hline Doxycycline & 60 & 20 & 7 & 3 \\
\hline Levofloxacin & 50 & 30 & 3 & 7 \\
\hline Chloramphenicol & 40 & 40 & 8 & 2 \\
\hline Cefoxitin & 40 & 40 & 6 & 4 \\
\hline Gatifloxacin & 50 & 30 & 3 & 7 \\
Ofloxacin & 60 & 20 & 3 & 7 \\
Ciprofloxacin & 50 & 30 & 6 & 4 \\
\hline Gentamycin & 60 & 20 & 8 & 2 \\
\hline Pristanamycin & 40 & 40 & 8 & 2 \\
\hline Tobramycin & 70 & 10 & 9 & 1 \\
\hline Netilmycin & 60 & 20 & 4 & 6 \\
Erythromycin & 50 & 30 & 6 & 4 \\
\hline Penicillin & 60 & 20 & 3 & 7 \\
\hline Co-Trimoxazole & - & - & 4 & 6 \\
\hline Ampicillin & 38 & 42 & 4 & 6 \\
\hline High strength & - & - & 1 & 9 \\
\hline Streptomycin & & & & \\
\hline High strength & - & - & 5 & 5 \\
\hline Gentamycin & & & & \\
\hline Vancomycin & - & - & 4 & 6 \\
\hline
\end{tabular}

\section{$\mathrm{S}=$ sensitive}

$\mathrm{R}=$ resistant

$\mathrm{n}=$ number of organisms

Table 3: Antibiotic sensitivity pattern of gram negative isolates

\begin{tabular}{|c|c|c|c|c|c|c|c|c|c|c|}
\hline \multirow[t]{3}{*}{ Antibiotics } & \multicolumn{10}{|c|}{ ORGANISMS $(n=140)$} \\
\hline & \multicolumn{2}{|c|}{$\begin{array}{c}\text { Escherichia oli } \\
\quad(n=10)\end{array}$} & \multicolumn{2}{|c|}{$\begin{array}{c}\text { Pseudomonas } \\
\text { aeruginosa }(n=70)\end{array}$} & \multicolumn{2}{|c|}{$\begin{array}{c}\text { Klebsiella } \\
\text { species. }(n=10)\end{array}$} & \multicolumn{2}{|c|}{$\begin{array}{c}\text { Citrobacter species } \\
(n=20)\end{array}$} & \multicolumn{2}{|c|}{$\begin{array}{l}\text { Proteus spp. } \\
\quad(n=30)\end{array}$} \\
\hline & S & $\mathbf{R}$ & S & $\mathbf{R}$ & $\mathbf{S}$ & $\mathbf{R}$ & $\mathbf{S}$ & $\mathbf{R}$ & $S$ & $\mathbf{R}$ \\
\hline Amikacin & 8 & 2 & 32 & 38 & 7 & 3 & 4 & 16 & 30 & 0 \\
\hline Fosfomycin & 8 & 2 & - & - & 2 & 8 & 8 & 12 & 28 & 2 \\
\hline Doxycycline & 4 & 6 & - & - & 10 & 0 & 7 & 13 & 30 & 0 \\
\hline Levofloxacin & 6 & 4 & 45 & 25 & 5 & 5 & 8 & 12 & 24 & 6 \\
\hline Cefoxitin & 6 & 4 & - & - & 6 & 4 & 15 & 5 & 22 & 8 \\
\hline Ofloxacin & 8 & 2 & - & - & 5 & 5 & 15 & 5 & - & - \\
\hline Ciprofloxacin & 10 & - & - & - & 2 & 8 & 10 & 10 & 5 & 25 \\
\hline Gentamycin & 8 & 2 & 58 & 12 & 4 & 6 & 10 & 10 & 21 & 9 \\
\hline Pristanamycin & - & - & 24 & 46 & - & - & - & - & - & - \\
\hline Co-Trimoxazole & 2 & 8 & - & - & 3 & 7 & 10 & 10 & 2 & 28 \\
\hline Ampicillin & 7 & 3 & - & - & 4 & 6 & 10 & 10 & 10 & 20 \\
\hline Cefotaxime & 7 & 3 & - & - & 6 & 4 & 16 & 4 & 18 & 12 \\
\hline Ceftazidime & 9 & 1 & - & - & 1 & 9 & 14 & 6 & 22 & 8 \\
\hline Ceftriaxone & 7 & 3 & - & - & 3 & 7 & 12 & 8 & - & - \\
\hline Cefepime & 2 & 8 & 66 & 4 & 2 & 8 & 13 & 7 & 14 & 16 \\
\hline Cefaclor & 4 & 6 & - & - & 4 & 6 & 16 & 4 & 15 & 15 \\
\hline Cefixime & 3 & 7 & - & - & 5 & 5 & 14 & 6 & - & - \\
\hline Cefuroxime & 7 & 3 & - & - & 5 & 5 & - & - & 5 & 15 \\
\hline Cefazolin & 8 & 2 & - & - & 6 & 4 & 12 & 8 & 13 & 17 \\
\hline Piperacillin & - & - & 47 & 23 & - & - & - & - & - & - \\
\hline Piperacillin-Tazobactum & - & - & 65 & 5 & - & - & - & - & - & - \\
\hline Aztreonam & - & - & 53 & 17 & - & - & - & - & - & - \\
\hline Colistin & - & - & 26 & 44 & - & - & - & - & - & - \\
\hline
\end{tabular}

$\mathrm{S}=$ sensitive

$\mathrm{R}=$ resistant

$\mathrm{n}=$ number of organisms 


\section{International Journal of Science and Research (IJSR) \\ ISSN (Online): 2319-7064}

Index Copernicus Value (2013): 6.14 | Impact Factor (2014): 5.611

\section{Discussion}

Ear discharge is one of the most frequently ordered samples for microbiological analysis in the study area. This indicates that ear infection is a common problem in the given area. In the present study, 400 specimens were included in the study, out of which 230 (67.5\%) showed positive bacterial growth and 170 were negative for any bacterial growth. Out of 90(39.1\%) isolates of Gram positive and 140 (60.8\%) were gram negative, which is compare to another previous study.[11,12,13]

In our study, commonest organisms isolated are Pseudomonas aeruginosa, Staphylococcus aureus and Proteus spp.. Less common organisms are E. coli and Klebsiella species. In this study Pseudomonas aeruginosa and Staphylococcus aureus were the most predominant organism being isolated in $70(30.4 \%)$ and $80(34.7 \%)$ of the cases. This was followed by E. coli in (4.3\%) cases. Proteus (13\%) and Klebsiella (4.3\%), Citrobacter spp. (8.6\%) and Enterococcus spp. (4.3\%). These findings were similar to those of similar studies.[15,16]

\section{Conclusion}

Pseudomonas aeruginosa and Staphylococcus aureus were the most common bacteria isolated from chronic discharging ears. Ciprofloxacin and gentamycin found to be the most sensitive against pseudomonas aeruginosa and Staphylococcus aureus. Development of resistance to commonly used antibiotics for treating Pseudomonas aeruginosa and Staphylococcus aureus infections in otitis media, alert us against indiscriminate uasage of antibiotics to prevent development of resistance.

\section{References}

[1] Variya A, Tainwala S, Mathur S: Bacteriology of acute otitis media in children. Indian J Med Microbiol. 2002, 20: 54-55.

[2] Woodfield G, Dugdale A, Evidence behind the WHO guidelines: hospital care for children: What is the most effective antibiotic regime for chronic suppurative otitis media in children?. J Tropical Pediatric. 2008, 54 (3): 151-156. View Article

[3] Akinpelu OV, Amusa YB, Komolafe EO, Adeolu AA, Oladele AO, Ameye SA: Challenges in management of chronic suppurative otitis media in a developing country. J Laryngol Otol. 2008, 122 (1): 16-20.View ArticlePubMed

[4] Bluestone CD, Klein JO: Microbiology. Otitis media in infants and children. Edited by: Bluestone CD, Klein JO. 2001, Philadelphia: P A W B. Saunders, 79-1014. 3

[5] Mymen Singh Med J. 2005 Jul;14(2):152-5.

[6] Kalcioglu,M.T. ; Ozturan,O. ; Durmaz, R. and Aktas,E. In vitro efficacy of the successive or staggered use of eardrops. Eur.Arch.Otorhinol. 2006; 263(5):395-398.

[7] Pendido Nde, O. ; Borni, A. ; Iha,L.C. ; Suguri,V.M. ; Onishi,E. Itracranial complica $\square$ ons of o $\square \square$ s media : 15 years experience in $33 \mathrm{pa} \square$ ents. Otolaryngol.Head Neck Surg. 2005: 132(1):37-42.
[8] Pajor,A. ; Durko,M. ; Jankowski,A. ; BartoszkoTyczkowska,A. and Stanczyk,R. Bacteriological evaluation in chronic o $\square \square \mathrm{s}$ media. Otolaryngol.Pol. 2006: 60(5):757-763.

[9] Hafidh,M.A. ; Keogh,I. ; Walsh,R.M. ; walsh,M. and Rawluk,D. Otogenic intracranial complica $\square$ ons: a 7 years retrospec $\square$ ve. Am.J.Otolaryngol. 2006: 27(6):390-395.

[10] Pakanati Siva Subba Rao, Mohiddin Shaik khaja . Indian Journal of Basic and Applied Medical Research; September 2014: Vol.-3, Issue- 4, P. 52-55.

[11]Abera B, Kibret M: Bacteriology and antimicrobial susceptibility of otitis media at dessie regional health research laboratory, Ethiopia. Ethiopian $J$ Health Develop. 2011, 25 (2): 161-167.

[12] Osazuwa F, Osazuwa E, Osime C, Igharo EA, Imade $P E$, Lofor $P$, Momoh $M$, Omoregie $R$, Dirisu J: Aetiologic agents of otitis media in Benin city, Nigeria. North Am J Med Sci. 2011, 3: 95-98.View Article

[13] Tesfaye $G$, Asrat D, Woldeamanuel $Y$, Gizaw M: Microbiology of discharging ears in Ethiopia. Asian Pacific J Tropical Med. 2009, 2 (1): 60-67.

[14] Iseh KR, Adegbite T: Pattern and bacteriology of acute suppurative otitis media in Sokoto, Nigeria. Ann Afri Med. 2004, 3 (4): 164-166.

[15]B.H.Senturia,C.D.Bluestone,J.O Klein et al., Ann.Otol.Rhinol.Laryngol.Suppl.1980,89,68,3.

[16] N.H.Moshi,B.M.Minja,East Afr Med J,2000,771,20. 